Case Report

\title{
Porcelain laminate veneer restorations bonded with a three- liquid silane bonding agent and a dual-activated luting composite
}

\author{
Hideo Matsumura ${ }^{1,2)}$, Yukiko Aida ${ }^{1)}$, Yumi Ishikawa ${ }^{3)}$ and Naomi Tanoue ${ }^{4)}$ \\ ${ }^{1)}$ Department of Fixed Prosthodontics, Nihon University School of Dentistry, Tokyo, Japan \\ ${ }^{2}$ Division of Advanced Dental Treatment, Dental Research Center, Nihon University School of Dentistry, \\ Tokyo, Japan \\ ${ }^{3)}$ Nihon University Graduate School of Dentistry, Tokyo, Japan \\ ${ }^{4)}$ Department of Specialized Dentistry, Nagasaki University Hospital of Medicine and Dentistry, \\ Nagasaki, Japan
}

(Received 20 October and accepted 6 November 2006)

\begin{abstract}
This clinical report describes the fabrication and bonding of porcelain laminate veneer restorations in a patient with anterior open spaces. Laminate veneer restorations made of feldspathic porcelain were etched with $5 \%$ hydrofluoric acid, rinsed under tap water, ultrasonically cleaned with methanol, and primed with a chemically activated three-liquid silane bonding agent (Clearfil Porcelain Bond). The enamel surfaces were etched with $40 \%$ phosphoric acid, rinsed with water, and primed with a two-liquid bonding agent (Clearfil New Bond) that contained a hydrophobic phosphate (10methacryloyloxydecyl dihydrogen phosphate; MDP). The restorations were bonded with a dual-activated luting composite (Clapearl DC). The veneers have been functioning satisfactorily for an observation period of one year. Combined use of the Clearfil bonding agents and Clapearl DC luting composite is an alternative to conventional materials for seating porcelain laminate veneer restorations, although the system is inapplicable to dentin bonding. (J. Oral Sci. 48, 261-266, 2006)
\end{abstract}

Keywords: acid; bonding; hydrofluoric acid; porcelain; silane.

Correspondence to Dr. Hideo Matsumura, Department of Fixed Prosthodontics, Nihon University School of Dentistry, 1-8-13 Kanda-Surugadai, Chiyoda-ku, Tokyo 101-8310, Japan

Tel: +81-3-3219-8145

Fax: +81-3-3219-8351

E-mail: matsumura@dent.nihon-u.ac.jp

\section{Introduction}

Over the last decade, bonding agents have been increasingly used for fixing porcelain laminate veneer restorations. This trend is mainly attributed to the development of surface preparation techniques for both the porcelain materials and tooth structure. Current bonding systems consist of both surface preparation techniques for mechanical retention and chemical bonding agents.

Etching feldspathic porcelain with hydrofluoric acid is effective in mechanically retaining resin-based bonding agents (1-5). Application of a methacrylate-based silane monomer enhanced the bonding between acrylic resin and feldspathic porcelain materials (6-9). The improvement in bonding of acrylic resin to enamel by phosphoric acid etching was reported more than 50 years ago (10). A hydrophobic phosphate monomer (11), 10 methacryloyloxydecyl dihydrogen phosphate (MDP), was also shown to effectively bond tooth structure (12). Based on the fact that both porcelain and enamel can be bonded with an appropriate bonding agent after etching, a luting system specially formulated for porcelain bonding (Clapearl, Kuraray Medical Inc., Tokyo, Japan) was introduced (13).

Due to the development as well as disappearance of numerous resin-based bonding systems within several years, it is difficult for clinicians to select a reliable system for seating tooth-colored restorations. The purpose of this report is to present a standardized fabrication and bonding technique for porcelain laminate veneer restorations applied 
to a patient with anterior open spaces.

\section{Case Report}

A 27-year old male patient presented with the chief complaint of dissatisfaction derived from unesthetic spacing between the maxillary anterior teeth (Fig. 1). On examination, there were no dental caries detected in the maxillary anterior dentition. Among the treatment options provided, the patient preferred insertion of four porcelain laminate veneer restorations with minimal reduction of enamel surfaces. Prior to treatment, diagnostic casts and wax models simulating the porcelain laminate veneers were prepared (Fig. 2), and consent to the treatment protocol was obtained from the patient.

The facial, mesial, and distal surfaces of the four maxillary incisors were minimally reduced with a highspeed diamond rotary instrument (ISO 199/014 and 199/016, Shofu Inc., Kyoto, Japan) under water coolant. The reduction was limited to within enamel (Figs. 3 and 4). Using a shade guide (Vita Lumin Vacuum, Vita Zahnfabrik, Bad Säckingen, Germany), shades A3.5 and B4 were selected for the patient. An impression of the maxillary dentition was made with silicone elastomeric materials (Exafine Putty and Injection, GC Corp., Tokyo, Japan) using a stock tray, and poured with die stone and lab stone (Fuji Rock and New Plastone, GC Corp.). Before mounting the working cast, an impression of the working cast was made with a silicone material (Fig. 5) and poured with refractory material (Fig. 6). Although the four anterior incisors were restored, the restoration on the left central incisor had to be re-fabricated. The laboratory and bonding procedures of this single restoration are presented here. Both the working and opposing casts were mounted on a semi-adjustable articulator (Spacy Articulator, YDM Corp., Tokyo, Japan) with an intercuspal position record (Fig. 7). The sagittal and lateral condylar paths were adjusted using three pieces of check-bite records.

A porcelain laminate veneer restoration was fabricated with feldspathic porcelain material (Vintage, Shofu Inc.), according to the manufacturer's instructions (Figs. 8-12). After try-in of the restoration, the surface to be bonded was etched with 5\% hydrofluoric acid (HF-Gel, GC Corp.) for 60 seconds (4), washed under tap water, ultrasonically cleaned in methanol, dried with an air syringe, and primed with a three-liquid ceramic bonding agent (Clearfil Porcelain Bond, Kuraray Medical Inc.) (7,9) (Figs. 13-18). The reduced enamel surface was etched with $40 \%$ phosphoric acid (K-Etchant, Kuraray Medical Inc.) for 30 seconds, washed, dried with an air syringe, and primed with a two-liquid bonding agent (Clearfil New Bond, Kuraray Medical Inc.) (Figs. 19-22).
The laminate veneer restoration was bonded with a dual-curable composite luting agent (Clapearl DC, Universal, Kuraray Medical Inc.) (Figs. 23-24). Lightpolymerization was performed with a hand-held unit (Quicklight, J. Morita Corp, Suita, Japan) for 60 seconds each on the incisal, mesial, and distal surfaces (Fig. 25). The marginal areas of the enamel-composite-porcelain interface were ground with a diamond rotary instrument and polished with a rotary silicone instrument filled with fine diamond particles (CompoMaster, Shofu Inc.). Figs. 26 and 27 show the post-operative view of the restorations. The patient was satisfied with the esthetics and function of the restoration after treatment. As part of the maintenance, the patient was recalled at 6-month intervals for reinforcement of oral hygiene and evaluation of oral function. The laminate veneer restorations have been functioning satisfactorily for 12 months (Figs. 28 and 29). Fig. 30 summarizes the surface preparation and bonding procedures of the restorations.

\section{Discussion}

Porcelain laminate veneer restorations were placed in a patient with maxillary anterior open spaces. All restorations were bonded with the Clearfil-Clapearl bonding system. The restorations have been functioning satisfactorily for over a year. As porcelain veneers were used, the restorative procedure could be performed without exposing dentin. This procedure is especially useful for patients with diastema, open spaces with low caries activity, and malformed teeth.

A feldspathic porcelain material (Vintage) was used in fabrication of the laminate veneer restorations. Vintage is a representative tooth-colored material suitable for both laminate veneer and porcelain-fused-to-metal restorations. When considerable modification of tooth color is required, another feldspar-based coloring porcelain (Lamina, Shofu Inc.) can be used in combination with Vintage. The Lamina porcelain is especially useful for laminate veneer restorations consisting of dentin with varying opacities. The advantages of using feldspathic porcelain include; 1) reproducibility of tooth color with a thin layer of the material, 2) low laboratory cost compared to other ceramic restorative systems, 3) excellent mechanical retentive characteristics after etching with hydrofluoric acid, and 4) excellent bonding characteristics with the use of appropriate silane bonding agents.

In the present case, mechanical retention was achieved by etching the porcelain with $5 \%$ hydrofluoric acid (4). The restorations were ultrasonically cleaned with methanol using a polyethylene cup. Ultrasonic cleaning after hydrofluoric acid etching effectively removes precipitates 


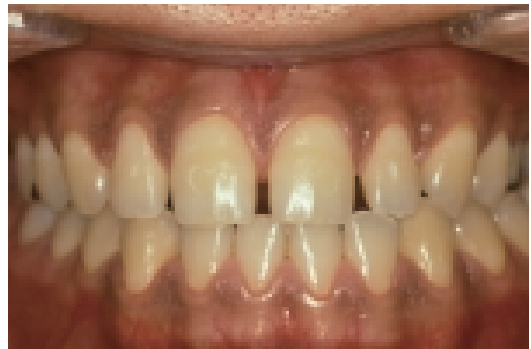

Fig. 1 Pre-operative view. Maxillary open spaces are evident.

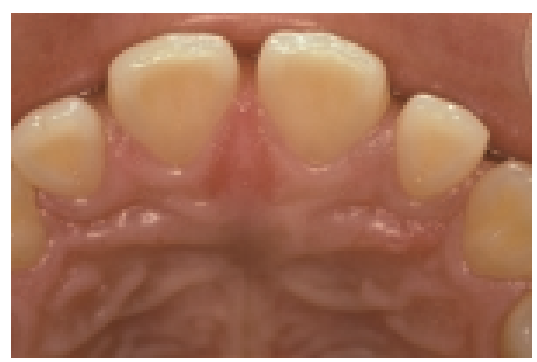

Fig. 4 Occlusal view. Reduction was limited to within enamel.

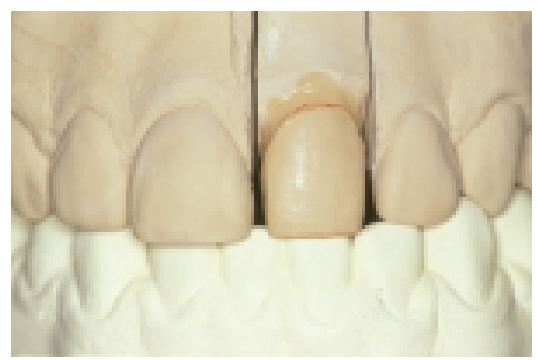

Fig. 7 A working cast and opposing cast mounted on an articulator.

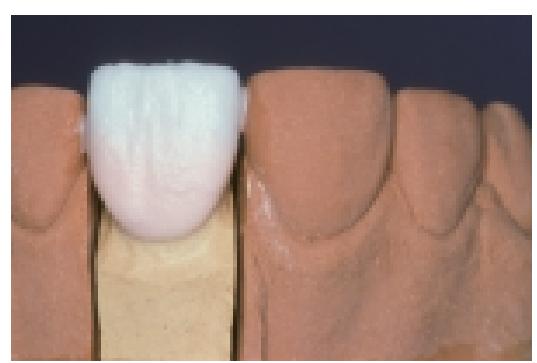

Fig. 10 Over-spread enamel porcelain material for compensation of firing shrinkage.

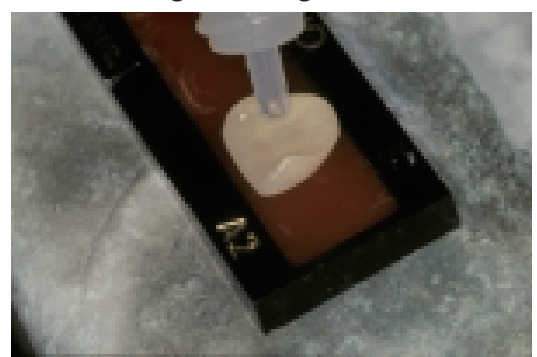

Fig. 13 Etching the porcelain with 5\% hydrofluoric acid gel for 60 seconds.

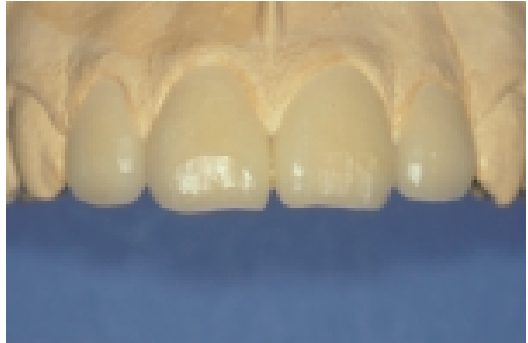

Fig. 2 A diagnostic cast and wax models simulating the porcelain laminate veneer restorations.

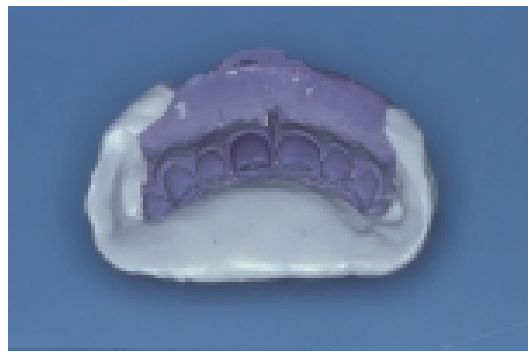

Fig. 5 Impression for refractory cast.

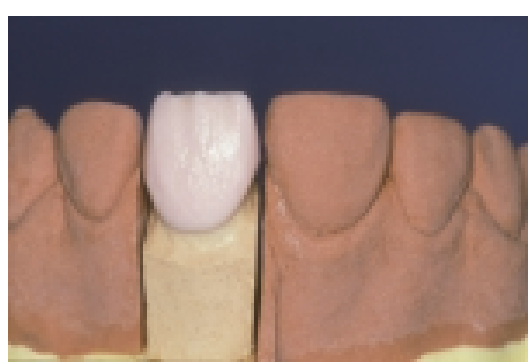

Fig. 8 Application of the primary layer of dentin porcelain.

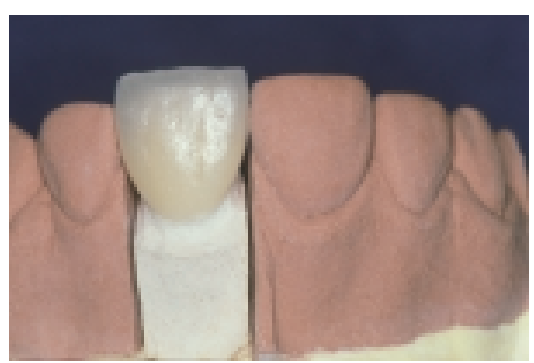

Fig. 11 Sintered restoration before glazing procedure.

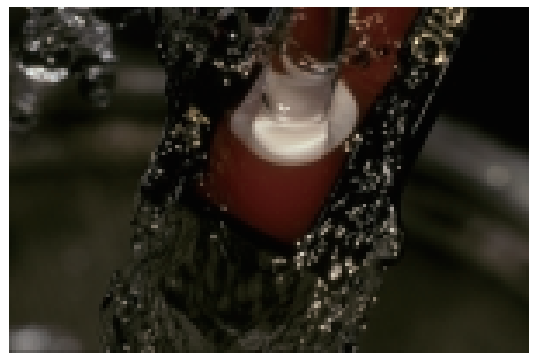

Fig. 14 Rinsing under tap water.

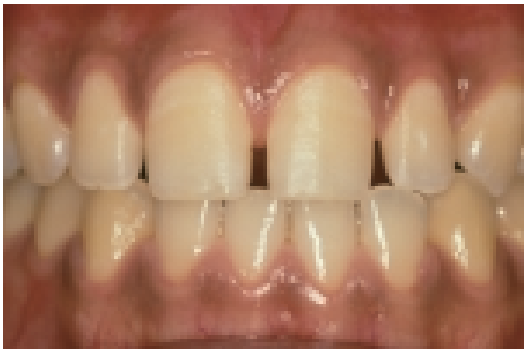

Fig. 3 Facial view after abutment preparation.

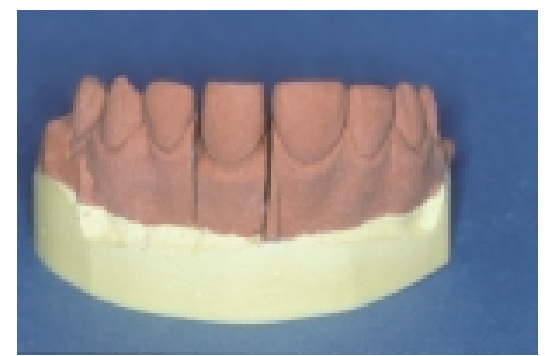

Fig. 6 Refractory cast to be used for porcelain firing procedure.

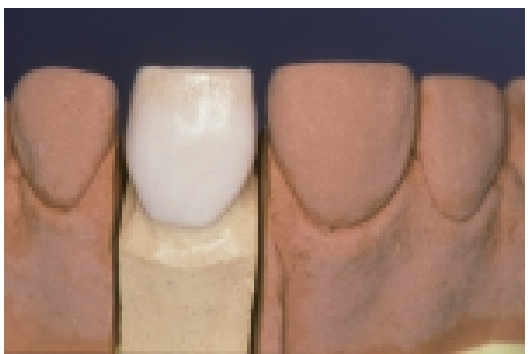

Fig. 9 Application of the secondary layer of porcelain with staining agent.

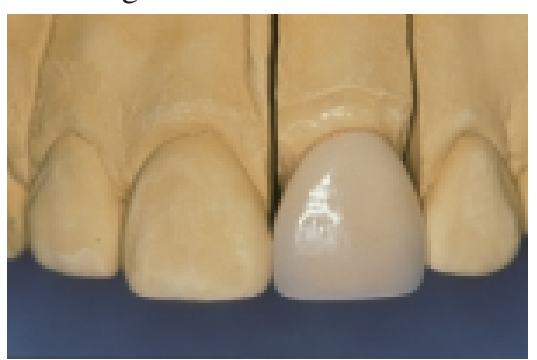

Fig. 12 A completed porcelain laminate veneer restoration on the working cast.

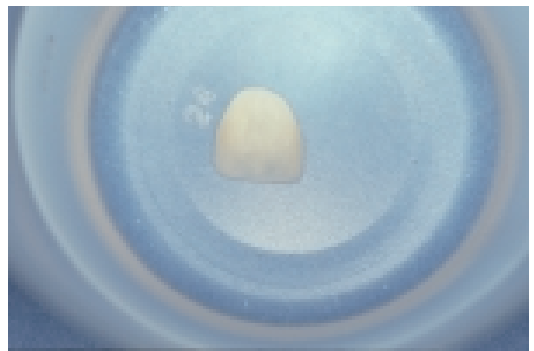

Fig. 15 Ultrasonic cleaning in a plastic cup with methanol. 


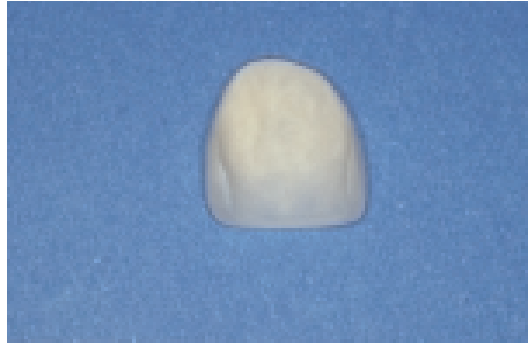

Fig. 16 Air-dried restoration.

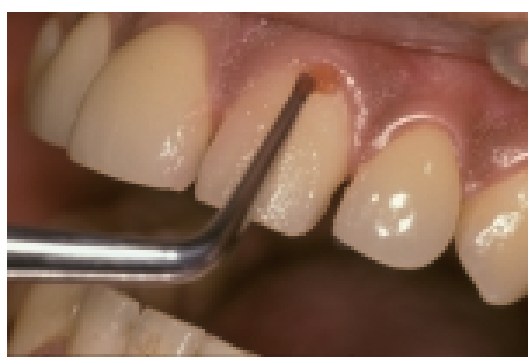

Fig. 19Etching enamel surface with $40 \%$ phosphoric acid gel for 30 seconds.

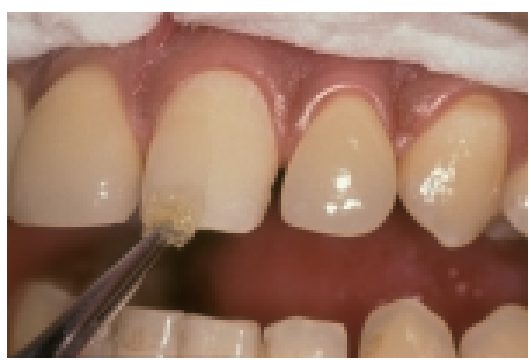

Fig. 22 Application of a two-liquid chemically-curable bonding agent (Clearfil New Bond).

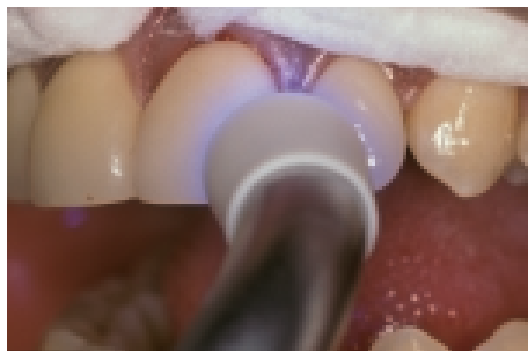

Fig. 25 Light curing with a hand-held unit.

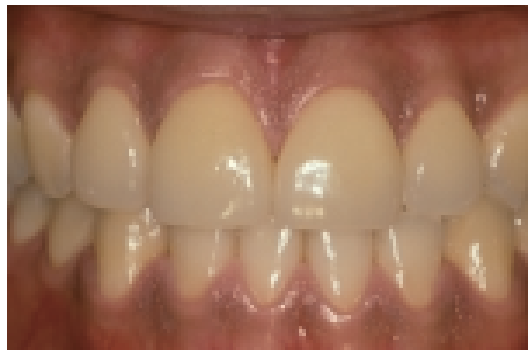

Fig. 28 Facial view of the restorations 12 months after seating.

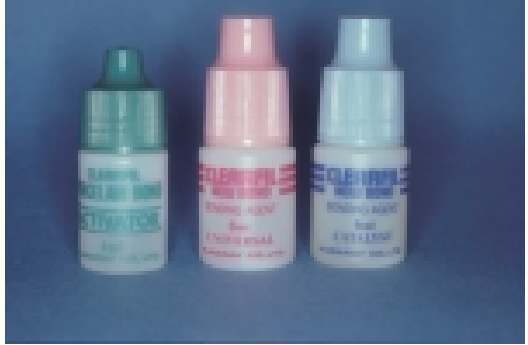

Fig. 17 A three-liquid porcelain bonding agent (Clearfil Porcelain Bond).

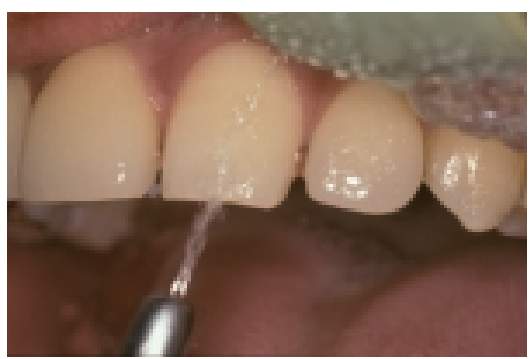

Fig. 20 Rinsing with water.

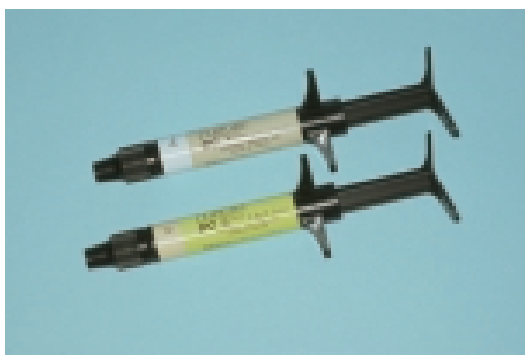

Fig. $23 \mathrm{~A}$ dual-polymerizable luting composite (Clapearl DC).

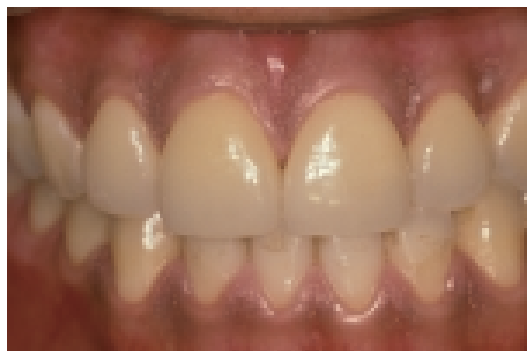

Fig. 26Facial view of the seated restorations.

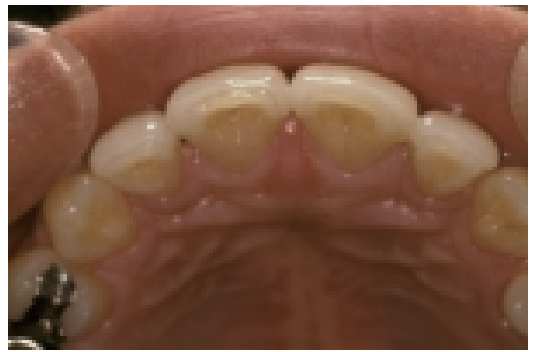

Fig. 29 Occlusal view of the restorations 12 months after seating.

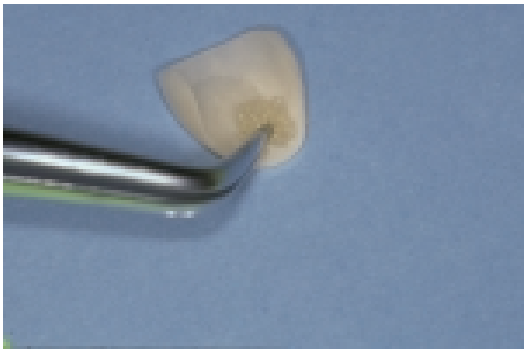

Fig. 18 Application of the three-liquid bonding agent with sponge pellet.

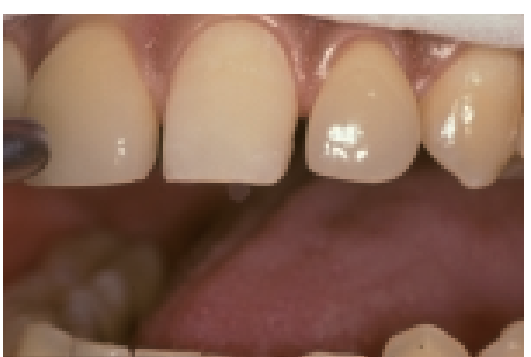

Fig. 21 Frosty appearance of etched enamel surface.

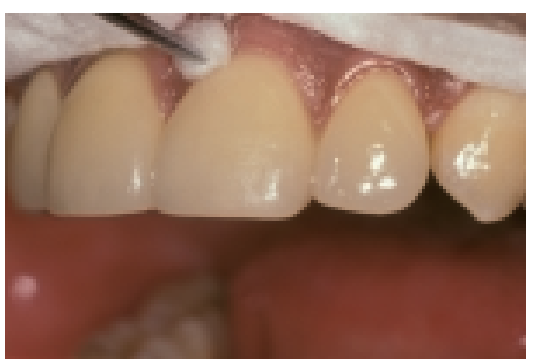

Fig. 24Removing excess luting material before light-exposure.

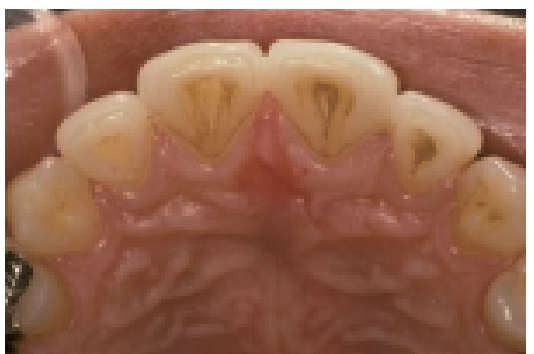

Fig. 27 Occlusal view of the seated restorations. 
and acid from the etched surface (14). Also, the water that penetrated onto the etched surface is probably replaced by methanol, which is more volatile than water. Thus, after air-drying, the wetting ability of the bonding agent may be enhanced in the case of etched and methanol-treated porcelain, compared to the etched and water-sprayed porcelain. The use of polymer cup during the ultrasonic cleaning is beneficial for the porcelain laminate veneer restorations, as it might reduce the possibility of microfracture in thin marginal areas of the brittle porcelain restorations. It is difficult to etch other ceramic materials such as alumina and zirconia with hydrofluoric acid; hence the mechanical retentive ability is inadequate.

The use of a methacrylate-based silane monomer is essential for bonding feldspathic porcelain. Based on previous reports evaluating in vitro bond strength $(9,15-$ 17), the three-liquid Clearfil Porcelain Bond material was selected for seating the restorations. The advantages of the Clearfil three-liquid system are that the acidic MDP monomer is effective for bonding tooth structure and for activating silane couplers, and the composition is selfpolymerizing. Application of this composition facilitates consistent bonding between the dual-curable luting agent and tooth structure.

$\begin{array}{lc}\text { Restoration } & \text { Enamel } \\ \text { Air-abrasion with alumina } & \\ \text { Removing refractory die material } & \\ \downarrow & \\ \text { Try-in } & \\ \downarrow & \\ \text { Porcelain etching } & \\ 5-10 \% \text { HF for } 60 \mathrm{~s} & \text { Enamel etching } \\ \downarrow & 37-40 \% \mathrm{H}_{3} \mathrm{PO}_{4} \text { for } 30 \mathrm{~s} \\ \text { Ultrasonic cleaning } & \downarrow \\ \text { Methanol or acetone } & \text { Rinsing with water spray } \\ \downarrow & \\ \text { Silane priming } & \\ \text { Clearfil Porcelain Bond } & \text { Application of bonding agent } \\ & \text { Clearfil New Bond } \\ & \downarrow \\ & \downarrow \\ & \text { Seating } \\ & \text { Clapearl DC } \\ & \downarrow \\ & \text { Removing excess luting agent } \\ & \downarrow \\ & \text { Light-exposure } \\ & \downarrow \\ & \text { Adjustment, Polishing }\end{array}$

Fig. 30 Surface preparation and bonding procedures for porcelain laminate veneer restorations.

\section{Acknowledgments}

This work was supported in part by grants from the Japan Dental Association, the Dental Research Center of Nihon University School of Dentistry, and a grant-in-aid (B 18390525) from the Japan Society for the Promotion of Science (JSPS).

\section{References}

1. Horn HR (1983) Porcelain laminate veneers bonded to etched enamel. Dent Clin North Am 27, 671-684

2. Calamia JR (1985) Etched porcelain veneers: the current state of the art. Quintessence Int 16, 5-12

3. Chen JH, Matsumura H, Atsuta M (1998) Effect of different etching periods on the bond strength of a composite resin to a machinable porcelain. J Dent 26, 53-58

4. Chen JH, Matsumura H, Atsuta M (1998) Effect of etchant, etching period, and silane priming on bond strength to porcelain of composite resin. Oper Dent 23, 250-257

5. Kato H, Matsumura H, Atsuta M (2000) Effect of etching and sandblasting on bond strength to sintered porcelain of unfilled resin. J Oral Rehabil 27, 103110

6. Paffenbarger GC, Sweeney WT, Bowen RL (1967) Bonding porcelain teeth to acrylic resin denture bases. J Am Dent Assoc 74, 1018-1023

7. Bertolotti RL, Lacy AM, Watanabe LG (1989) Adhesive monomers for porcelain repair. Int $\mathrm{J}$ Prosthodont 2, 483-489

8. Matsumura H, Kawahara M, Tanaka T, Atsuta M (1989) A new porcelain repair system with a silane coupler, ferric chloride, and adhesive opaque resin. J Dent Res 68, 813-818

9. Matsumura H, Kato H, Atsuta M (1997) Shear bond strength to feldspathic porcelain of two luting cements in combination with three surface treatments. J Prosthet Dent 78, 511-517

10. Buonocore MG (1955) A simple method of increasing the adhesion of acrylic filling materials to enamel surfaces. J Dent Res 34, 849-853

11. Omura I, Yamauchi J, Nagase Y, Uemura F (1983) Methacryloyloxyalkyl dihydrogen phosphate. Japan Pat Appl S58-21687 (in Japanese)

12. Fusayama A, Kohno A (1989) Marginal closure of composite restorations with the gingival wall in cementum/dentin. J Prosthet Dent 61, 293-296

13. Nakamura T, Miyamae M, Koh N, Hino T, Maruyama T (1992) Adhesive strength between teeth and resin cements for porcelain laminate veneer. J Osaka Univ Dent Sch 32, 21-26 
14. Canay S, Hersek N, Ertan A (2001) Effect of different acid treatments on a porcelain surface. J Oral Rehabil 28, 95-101

15. Kato H, Matsumura H, Tanaka T, Atsuta M (1996) Bond strength and durability of porcelain bonding systems. J Prosthet Dent 75, 163-168

16. Sato K, Matsumura H, Atsuta M (1999) Effect of three-liquid bonding agents on bond strength to a machine-milled ceramic material. J Oral Rehabil 26, 570-574

17. Ide T, Tanoue N, Yanagida H, Atsuta M, Matsumura $\mathrm{H}$ (2005) Effectiveness of bonding systems on bonding durability of a prefabricated porcelain material. Dent Mater J 24, 257-260 